\title{
Time-Fractional Fourier Law in a finite hollow cylinder under Gaussian-distributed heat flux
}

\author{
Slawomir Blasiak ${ }^{1, *}$ \\ ${ }^{1}$ Kielce University of Technology, Faculty of Mechatronics and Mechanical Engineering, \\ Department of Mechanical Technology and Metrology, Aleja Tysiaclecia Panstwa Polskiego 7, 25-314 Kielce, Poland
}

\begin{abstract}
This paper presents the solution of the theoretical model of heat conduction based on timefractional Fourier equation for a finite hollow cylinder treated with heat flux on one of the front surfaces. A derivative of fractional order in the Caputo sense was applied to record the temperature derivative in time. The distributions of temperature fields in the hollow cylinder were determined with the use of Fourier-Bessel series, as surface functions of two variables $(r, \theta)$. The distributions of temperature fields were determined using analytical methods and applying integral transformation methods. The Laplace transform with reference to time, the Fourier finite cosine transform with reference to axial coordinate $z$ and Marchi-Zgrablich transform for radial coordinate $r$. The fractional heat conduction equation was analysed for $0<\alpha \leq 2$.
\end{abstract}

\section{Introduction}

The research papers concerning heat conduction in structural elements in machines and devices are based on the classical Fourier's law. They might be generally classified e.g. in terms of the shape of the medium where the heat conduction is considered. Most research papers describe heat conduction in axial-symmetrical elements: rods, cylinders, plates of finite or infinite length or thickness [1-3]. There are as well studies which include discussions concerning heat conduction in relation to thermo-elastic deformations in different types of mechatronic devices [4-7]. The classical theory of heat conduction is based on the Fourier law, which might be recorded in the following form:

$$
\mathbf{q}=-\lambda \operatorname{grad}(\vartheta)
$$

where $\mathbf{q}$ is the heat flux vector, $\vartheta=T-T_{o}$ denotes change in temperature, and $\lambda$ is the thermal conductivity. In combination with the law of conservation of energy is expressed locally by:

$$
-\nabla \mathbf{q}(t)=\rho C_{p} \frac{\partial \vartheta(t)}{\partial t}
$$

where $\rho$ is density and $C_{p}$ is the specific heat capacity at constant pressure.

Another aspect of the research papers which has gained in popularity over the last thirty years is differential and integral calculus conducted on derivatives of fractional order [8-16]. The differential calculus (and integral calculus) of fractional order is a natural extension of the following concepts: integral and differential included in traditional differential and integral calculus. The interest of a large number of scientists is caused by numerous observations which demonstrate that a lot of phenomena might be explained in a more detailed way and modelled using the above mentioned differential calculus of fractional order [17]. The most crucial examples include the application of the differential calculus of fractional order to build controllers, such as $\mathrm{PI}^{\lambda} \mathrm{D}^{\mu}$, model the processes of heating and heat conduction, thermo-elasticity, thermal shock [18, 19], complex nonlinear systems, super capacitors, electrical systems, electrical filters, phenomena of dielectric relaxation, phenomena of diffusion and viscoelastic phenomena. The time fractional heat conduction equation might be recorded in the following form:

$$
\frac{\partial^{\alpha} \vartheta}{\partial t^{\alpha}}=\kappa \Delta \vartheta \quad 0<\alpha \leq 2,
$$

where $\kappa=\frac{K}{\rho C_{p}}$

The Caputo fractional derivative is expressed with the dependency:

$$
\begin{aligned}
\frac{\partial^{\alpha} f(t)}{\partial t^{\alpha}} & \equiv D_{C}^{\alpha} f(t)=I^{n-\alpha} D^{n} f(t) \\
& =\frac{1}{\Gamma(n-\alpha)} \int_{0}^{t}(t-\tau) \quad \frac{d^{n} f(\tau)}{d \tau^{n}} d \tau \\
t>0, \quad n-1<\alpha<n &
\end{aligned}
$$

With reference to the subject of this paper, the results of heat conduction in the hollow cylinder under Gaussiandistributed heat flux were presented.

\footnotetext{
*Corresponding author: sblasiak@tu.kielce.pl
} 


\section{The boundary and initial conditions for heat conduction}

In order to solve the equation describing the temperature distribution in the hollow cylinder, it is necessary to introduce initial and boundary conditions. On the surface of the hollow cylinder, the initial conditions were applied which were presented graphically in Fig. 1.

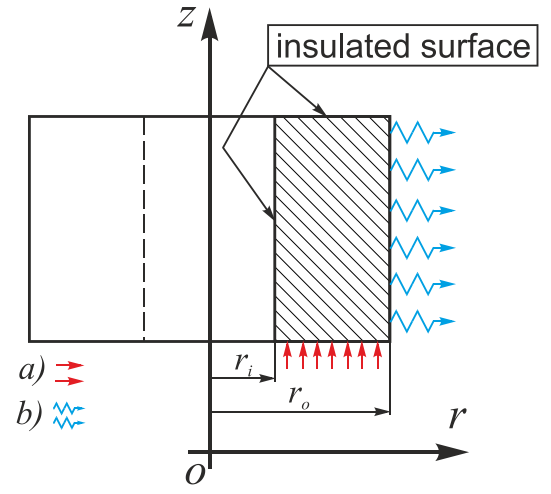

Fig. 1. Boundary conditions for heat transfer in the hollow cylinder. a) heat flux, b) convection.

It was assumed that the cylindrical surface on the inner radius $r_{i}$ and the top surface of the cyliner are completely isolated from the surrounding which takes the general form:

$$
\frac{\partial \vartheta}{\partial n}=0 .
$$

For the cylinder surface (on the outer radius $r_{o}$ ) which has a direct contact with the surrounding medium, the heat is transferred via convection, which was recorded as:

$$
-\left.\lambda \frac{\partial \vartheta}{\partial r}\right|_{r=r_{o}}=\left.\alpha^{f} \vartheta\right|_{r=r_{o}} .
$$

where $\alpha^{f}$ is the heat transfer coefficient. It was assumed that the lower surface of the hollow cylinder is exposed to heat flux expressed with the dependency:

$$
\varphi_{q}=\frac{1200}{\sigma \sqrt{2 \pi}} \exp \left(-\frac{(r-\bar{r})^{2}}{2 \sigma^{2}}\right)
$$

where $\sigma=0.0005$, whereas $\bar{r}=r_{i}+0.5\left(r_{o}-r_{i}\right)$. The heat flux distribution on the lower surface of the hollow cylinder was presented in Fig. 2.Next chapter presents the analytical solution of the above presented issue.

\section{Analytical solution}

By adopting a cylindrical coordinate system consistent with the geometry of the physical model in question, the equation (3) might be recorded in the following form:

$$
\frac{\partial^{\alpha} \vartheta}{\partial \tau^{\alpha}}=\frac{1}{r} \frac{\partial \vartheta}{\partial r}+\frac{\partial^{2} \vartheta}{\partial r^{2}}+\frac{\partial^{2} \vartheta}{\partial z^{2}}
$$

It was adopted that $\tau=\kappa t$. To solve the equation (8), Marchi-Zgrablich transform, Fourier finite cosine transform and the Laplace transform for time-fractional differential equations were applied.

The general form of Marchi-Zgrablich integral transform might be defined in the following way:

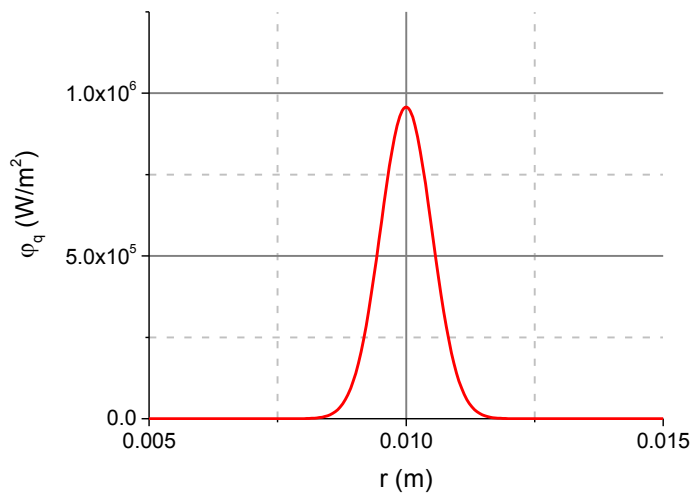

Fig. 2. The heat flux distribution on the lower surface of the hollow cylinder.

$$
\mathrm{H}(f(x))=\bar{f}(n)=\int_{0}^{r_{o}} r f(x) S_{p}\left(\lambda, \alpha, k_{n} r\right) d r
$$
form:

The reverse integral transform takes the following

$$
\begin{gathered}
\mathrm{H}^{-1}(\bar{f}(x))=f(x)=\sum_{n} a_{n} S_{p}\left(\lambda, \alpha, k_{n} r\right) \\
\text { where: } \\
a_{n}=\frac{\bar{f}_{p}(n)}{C_{n}} \\
C_{n}=\int_{r_{n}}^{r_{0}} r\left[S_{p}\left(\lambda, \alpha, k_{n} r\right)\right]^{2} d r \\
\mathrm{H}^{-1}(\bar{f}(x))=f(x)= \\
=\sum_{n} \frac{\overline{f_{p}}(n)}{\int_{r}^{r_{0}} r\left[S_{p}\left(\lambda, \alpha, k_{n} \cdot r\right)\right]^{2} d r} S_{p}\left(\lambda, \alpha, k_{n} r\right)
\end{gathered}
$$

The equation (8) takes then the following form:

$$
\frac{\partial^{\alpha} \bar{\vartheta}}{\partial \tau^{\alpha}}=\left(\frac{\partial^{2} \bar{\vartheta}}{\partial z^{2}}-k_{n}{ }_{n}^{2} \bar{\vartheta}\right)
$$

Fourier finite cosine transform is defined as follows:

$$
\mathrm{J}_{c}[g(x)]=g^{*}(n)=\int_{0}^{L} g(x) \cos \left(\frac{n \pi x}{L}\right) d x
$$

Where $n=1,2,3, \ldots$

$$
\begin{gathered}
J_{c}\left[\frac{d^{2} g(x)}{d x^{2}}\right\rceil=\left.(-1)^{n} \frac{d g(x)}{d x}\right|_{x=L}-\left.\frac{d g(x)}{d x}\right|_{x=0}+ \\
-\frac{n^{2} \pi^{2}}{L^{2}} g^{*}(n)
\end{gathered}
$$

The reverse transform is described by:

$$
\begin{aligned}
& \mathrm{J}_{c}^{-1}\left[g^{*}(n)\right]=g(x)=\frac{g^{*}(n=0)}{L}+ \\
& +\frac{2}{L} \sum_{n=1}^{\infty} g^{*}(n) \cos \left(\frac{n \pi x}{L}\right)
\end{aligned}
$$

By applying Fourier finite cosine transform to particular elements of the equation (14) with regard to the dependency:

$$
\begin{gathered}
J_{c}\left[\frac{\partial^{2} \bar{\vartheta}}{\partial z^{2}}\right]=\left.(-1)^{n} \frac{d}{d z}\right|_{z=n}-\left.\frac{d \bar{\vartheta}}{d z}\right|_{z=0}-\frac{n^{2} \pi^{2}}{L^{2}} \bar{\vartheta}^{*}(n)= \\
=\frac{\varphi_{q}}{\lambda}-\frac{n^{2} \pi^{2}-\bar{\vartheta}^{*}}{L^{2}}(n) \\
J_{c}[\bar{\vartheta}]=\bar{\vartheta}^{*}(n)=\int_{0}^{L} \bar{\vartheta}(x) \cos \left(\frac{n \pi x}{L}\right) d x
\end{gathered}
$$


The following was achieved:

$$
\frac{\partial^{\alpha} \bar{\vartheta}^{*}}{\partial \tau^{\alpha}}=\left(\frac{\overline{\varphi_{q}}}{\lambda}-\frac{n^{2} \pi^{2} \bar{\vartheta}^{*}}{L^{2}}(n)-k^{2}{ }_{n} \bar{\vartheta}^{*}(n)\right)
$$

The dependence (8) describes the time-fractional heat conduction equation, for which the following initial conditions were adopted.

$$
\begin{aligned}
& t=0 \quad \vartheta=0, \quad 0<\alpha \leq 2, \\
& t=0 \quad \frac{\partial \vartheta}{\partial t}=0, \quad 1<\alpha \leq 2 .
\end{aligned}
$$

as well as the boundary conditions for the area of the ring cross-section.

The left side in the equation (8) is the Caputo frictional derivative. Using the Laplace transform rule in the general form:

$$
\mathrm{L}\left\{D_{c}^{\alpha} f(t)\right\}=s^{\alpha} \hat{f}(s)-\sum_{k=0}^{n-1} f^{(k)}\left(0^{+}\right) s^{\alpha-1-k}, n-1<\alpha<n
$$

the equation (8) took the following form:

$$
s^{\alpha} \hat{\vartheta}^{*}(s)=\frac{\overline{\varphi_{q}}}{\lambda s}-\frac{m^{2} \pi^{2}}{L^{2}} \hat{\vartheta}^{*}(s)-k_{n}^{2} \hat{\vartheta}^{*}(s)
$$

By applying subsequent algebraic transformations, the following was achieved:

$$
\hat{\vartheta}^{*}(s)=\frac{\bar{\varphi}_{q}}{\lambda} \frac{1}{\omega^{2}}\left(\frac{1}{s}-\frac{s^{\alpha-1}}{\left(s^{\alpha}+\omega^{2}\right)}\right)
$$

Where $\left(\frac{m^{2} \pi^{2}+L^{2} k_{n}^{2}}{L^{2}}\right)=\omega^{2}$

After using the reverse Laplace transform, the dependence (24) takes the following form:

$$
\begin{aligned}
\bar{\vartheta}^{*}(s) & =\frac{\bar{\varphi}_{q}}{\lambda \omega^{2}}\left(1-E_{\alpha}\left(-\kappa \omega^{2} t^{\alpha}\right)\right)= \\
& =\frac{\bar{q}}{\lambda}\left(\frac{1}{\omega^{2}}-\frac{1}{\omega^{2}} E_{\alpha}\left(-\kappa \omega^{2} t^{\alpha}\right)\right)
\end{aligned}
$$

Where $E_{\alpha, \beta}(z)$ is Mittag-Leffler type function defined by the series $[20,21]$ :

$$
E_{\alpha, \beta}(z)=\sum_{v=1}^{\infty} \frac{z^{v}}{\Gamma(\alpha v+\beta)} \quad \alpha>0, \quad \beta>0
$$

After applying two subsequent integral transforms, the equation describing the temperature distribution in the hollow cylinder takes its final form:

$$
\begin{aligned}
& \vartheta=\frac{1}{L} \sum_{n=1}^{\infty} \frac{\int_{r_{i}}^{r_{i}} r \bar{\varphi}_{q} S_{p}\left(\lambda^{s}, \alpha^{f}, k_{n} r\right) d r}{\lambda \int_{r_{i}}^{r_{o}} r\left[S_{p}\left(\lambda, \alpha, k_{n} r\right)\right]^{2} d r} . \\
& S_{p}\left(\lambda, \alpha, k_{n} r\right) \sum_{m=1}^{\infty} \underbrace{(\underbrace{2}-\frac{1}{\omega^{2}}-\frac{1}{\omega^{2}} E_{\alpha}\left(-\kappa \omega^{2} t^{\alpha}\right))+}_{\omega_{0}}+-- \\
& +\frac{2}{L} \sum_{n=1}^{\infty} \frac{\int_{i} r \bar{\varphi}_{q} S_{p}\left(\lambda^{s}, \alpha^{f}, k_{n} r\right) d r}{\lambda \int_{r_{i}}^{r_{0}} r\left[S_{p}\left(\lambda, \alpha, k_{n} r\right)\right]^{2} d r} . \\
& S_{p}\left(\lambda, \alpha, k_{n} r\right) \sum_{m=1}^{\infty} \cos \left(\frac{m \pi x}{L}\right)\left(\frac{1}{\omega^{2}}-\frac{1}{\omega^{2}} E_{\alpha}\left(-\kappa \omega^{2} t^{\alpha}\right)\right)
\end{aligned}
$$

When $\alpha=1$, the equation (27) might be recorded as:

$$
\begin{aligned}
& \vartheta=\frac{1}{L} \sum_{n=1}^{\infty} \frac{\int_{r_{i}}^{r_{0}} r \bar{\varphi}_{q} S_{p}\left(\lambda^{s}, \alpha^{f}, k_{n} r\right) d r}{\lambda \int_{r_{i}}^{r_{0}} r\left[S_{p}\left(\lambda, \alpha, k_{n} r\right)\right]^{2} d r} . \\
& S_{p}\left(\lambda, \alpha, k_{n} r\right) \sum_{m=1}^{\infty} \underbrace{\left(\frac{1}{\omega^{2}}-\frac{1}{\omega^{2}} \exp \left(-\kappa \omega^{2} t^{\alpha}\right)\right)}_{\omega^{\prime}}+ \\
& +\frac{2}{L} \sum_{n=1}^{\infty} \frac{\int_{\omega_{i}}^{r_{i}} r \bar{\varphi}_{q} S_{p}\left(\lambda^{s}, \alpha^{f}, k_{n} r\right) d r}{\lambda \int_{r_{i}}^{r_{0}} r\left[S_{p}\left(\lambda, \alpha, k_{n} r\right)\right]^{2} d r} . \\
& S_{p}\left(\lambda, \alpha, k_{n} r\right) \sum_{m=1}^{\infty} \cos \left(\frac{m \pi x}{L}\right)\left(\frac{1}{\omega^{2}}-\frac{1}{\omega^{2}} \exp \left(-\kappa \omega^{2} t^{\alpha}\right)\right)
\end{aligned}
$$

\section{RESULTS AND DISCUSSION}

The parameters characterising the material used for the hollow cylinder are presented in the table below.

Table 1. Properties of the Carbon Steels material

\begin{tabular}{|c|c|c|c|}
\hline $\begin{array}{c}\text { Density } \rho \\
\left(10^{3} \mathrm{~kg} / \mathrm{m}^{3}\right)\end{array}$ & 7.85 & $\begin{array}{c}\text { Thermal } \\
\text { Expansion } \tau \\
\left(10^{-6} / \mathrm{K}\right)\end{array}$ & 11 \\
\hline $\begin{array}{c}\text { Young's } \\
\text { modulus } \\
\mathrm{E}(\mathrm{GPa})\end{array}$ & 190 & $\begin{array}{c}\text { Thermal } \\
\text { conductivity } \lambda \\
(\mathrm{W} / \mathrm{mK})\end{array}$ & 24.3 \\
\hline $\begin{array}{c}\text { Poisson ratio } \\
v\end{array}$ & 0.27 & $\begin{array}{c}\text { Specific Heat } \mathrm{C}_{\mathrm{p}} \\
(\mathrm{J} / \mathrm{kgK})\end{array}$ & 450 \\
\hline
\end{tabular}

The characteristic dimensions of the hollow cylinder as well as the reference operating parameters were summarized in Table 2. It was assumed that the studied cylinder was surrounded with fluid $T_{o}$ of $20^{\circ} \mathrm{C}$.

The presented solution of the mathematical model makes it possible to demonstrate the obtained results for two cases:

$>$ The first case which might be called the classical one, when $\alpha=1$.

$>$ The second case when $\alpha$ in the equation (8) takes the value of incomplete order $0<\alpha \leq 2$.

In this part of this paper, the results of the simulation studies concerning the influence of geometric and operating parameters on the temperature distribution in the ring were collected.

Table 2. Geometry- and performance-related parameters

\begin{tabular}{|c|c|}
\hline \multicolumn{2}{|c|}{ Geometry-related parameters } \\
\hline Inner radius $r_{i}(\mathrm{~m})$ & 0.005 \\
\hline Outer radius $r_{o}(\mathrm{~m})$ & 0.015 \\
\hline Thickness of the hollow cylinder $h(\mathrm{~m})$ & 0.015 \\
\hline Performance-related parameters \\
\hline Heat transfer coefficient $($ water $) \alpha^{f}\left(\mathrm{~W} / \mathrm{m}^{2} \mathrm{~K}\right)$ & 1200 \\
\hline Outer Fluid temperature $T_{o}\left({ }^{\circ} \mathrm{C}\right)$ & 20 \\
\hline
\end{tabular}



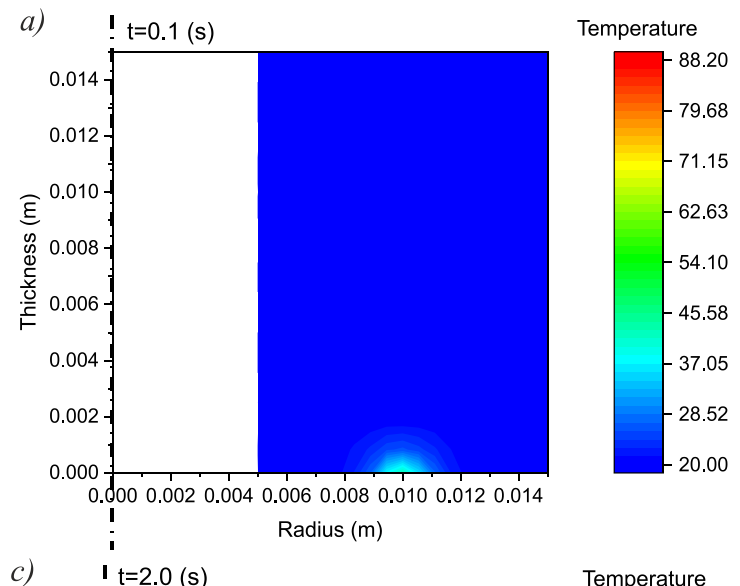

c)

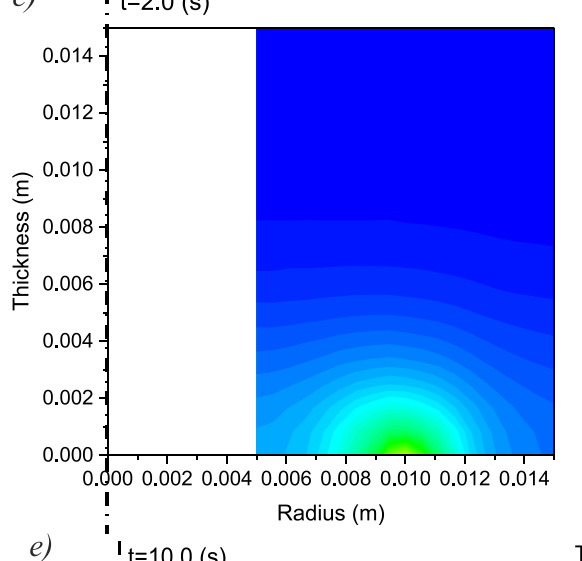

Temperature
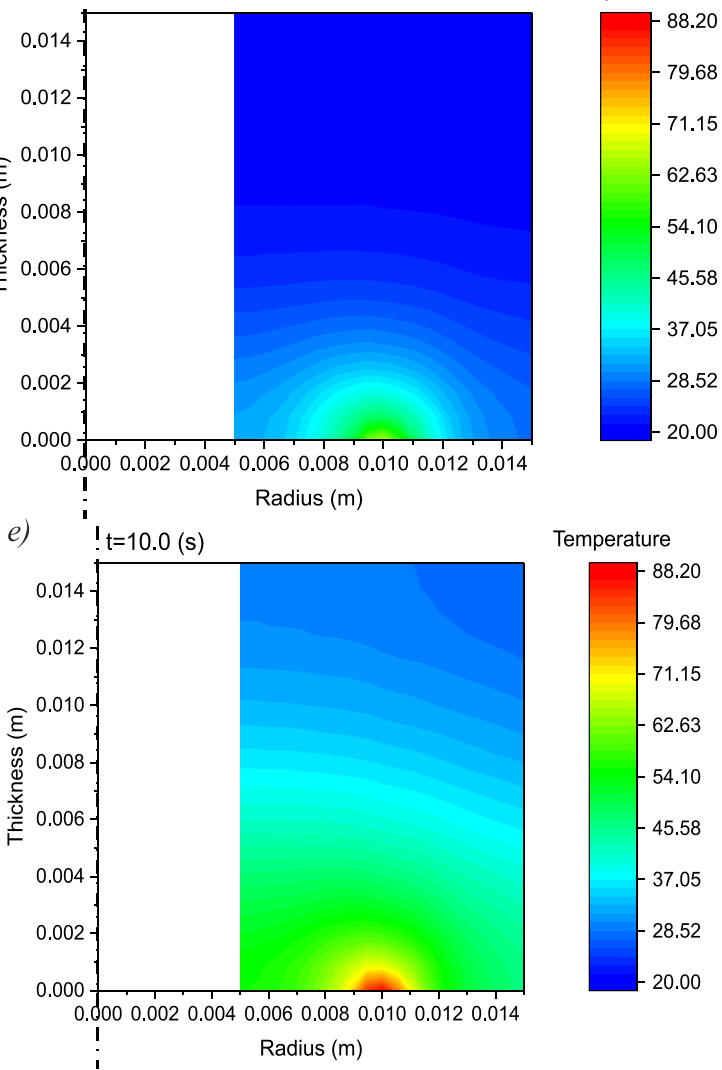
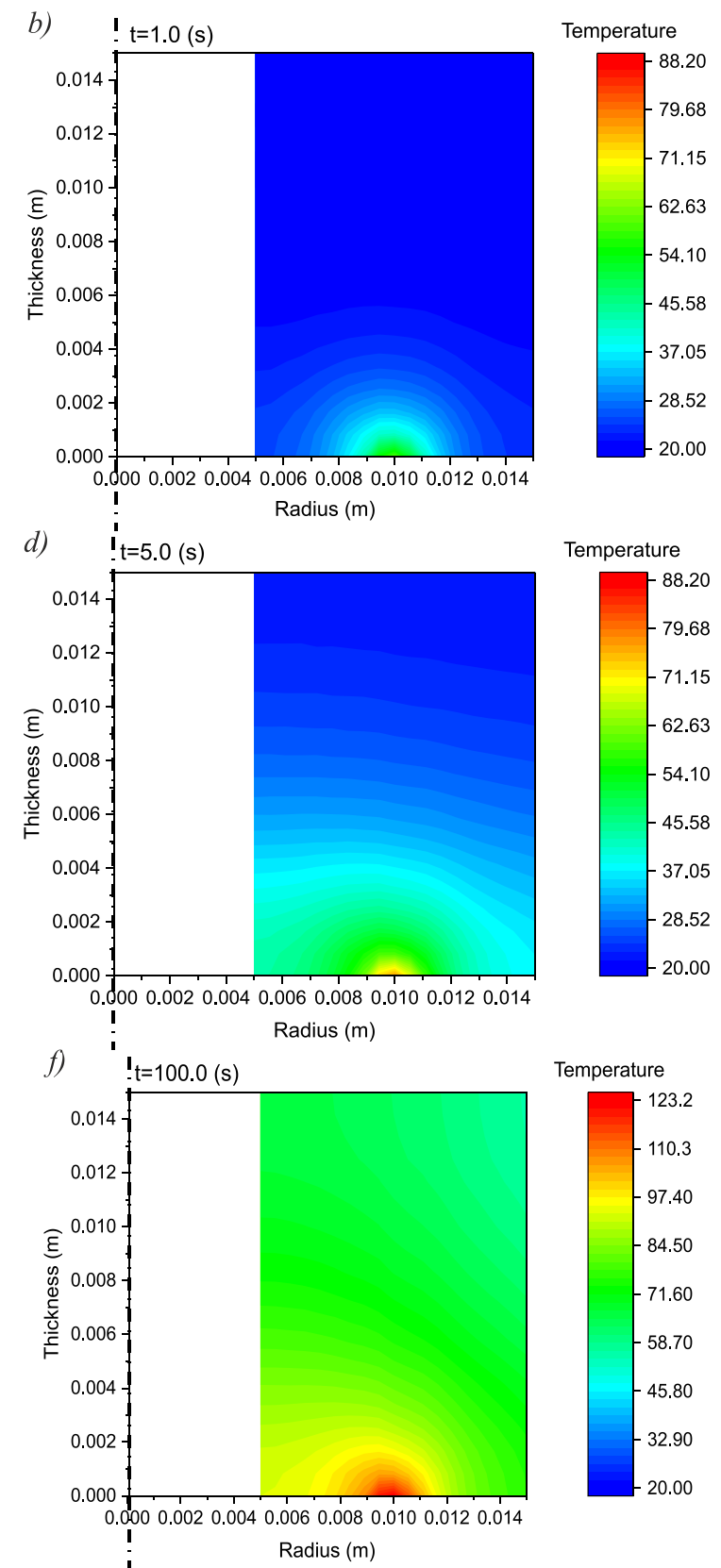

Fig. 3. The temperature distribution in the cross-section of the hollow cylinder for different $t$.

The temperature changes were presented graphically as temperature distributions for the cross section of the hollow cylinder. and Fig. 4 present the temperature changes for the simulation time within the range $0.1-100(s)$. The results presented in the graphs below were developed for $\alpha=1$, i.e. for the classical solution of the Fourier equation (see eq. (28)).shows the process of heating the studied element with heat flux. The maximum temperature value is located on the radius $\bar{r}$, i.e. in the place with the highest density of the heat flux.

When $\alpha=1$ and when $t \rightarrow \infty$, then the temperature $\vartheta$ tends to achieve steady state temperature. Such a state in the physical model in question takes place after approximately $50(\mathrm{~s})$.

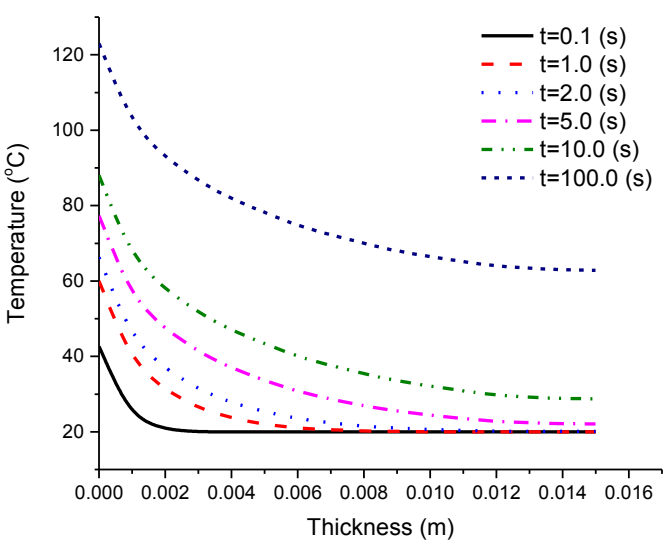

Fig. 4. The temperature distribution along the ring thickness on the radius $\bar{r}$ for different time values $\mathrm{t}$. 
a)

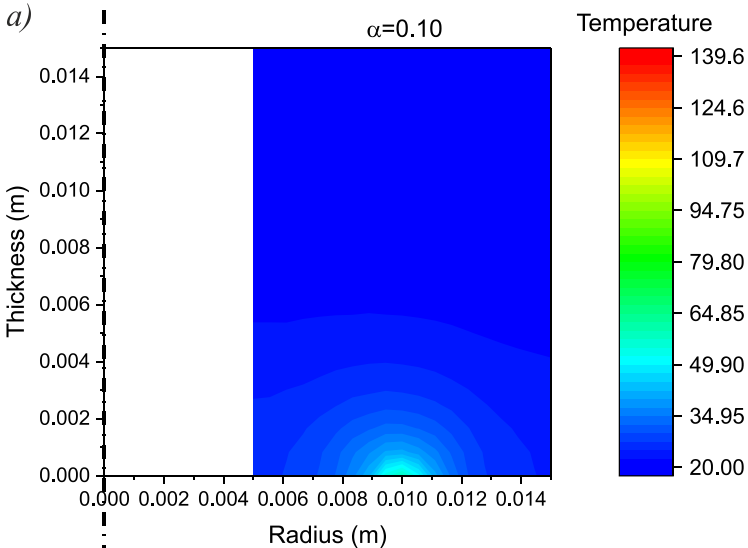

c)
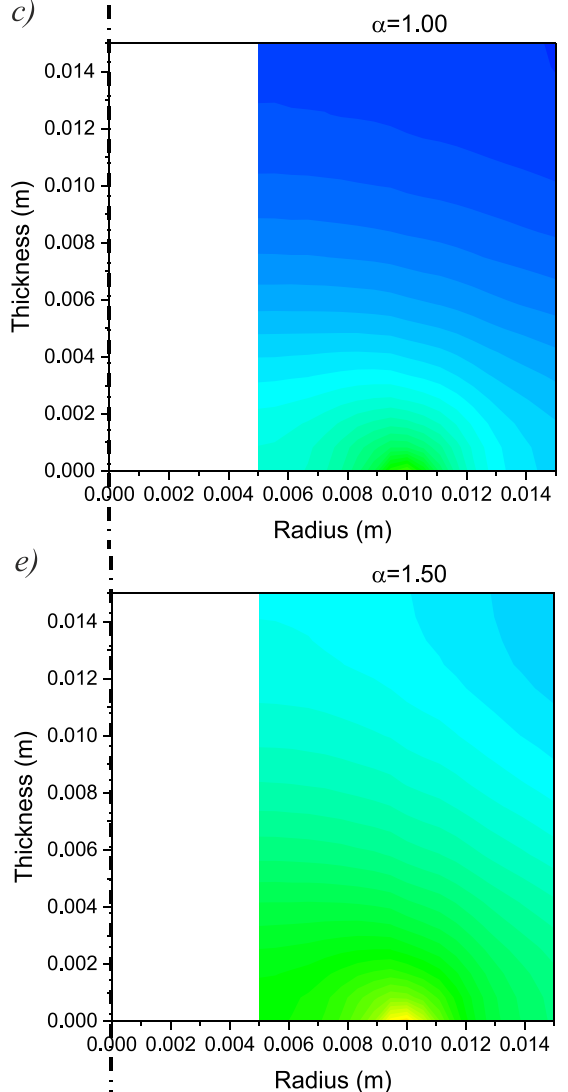

Temperature

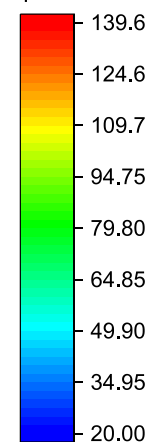

Temperature

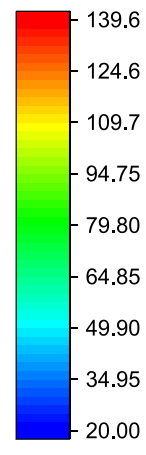

b)
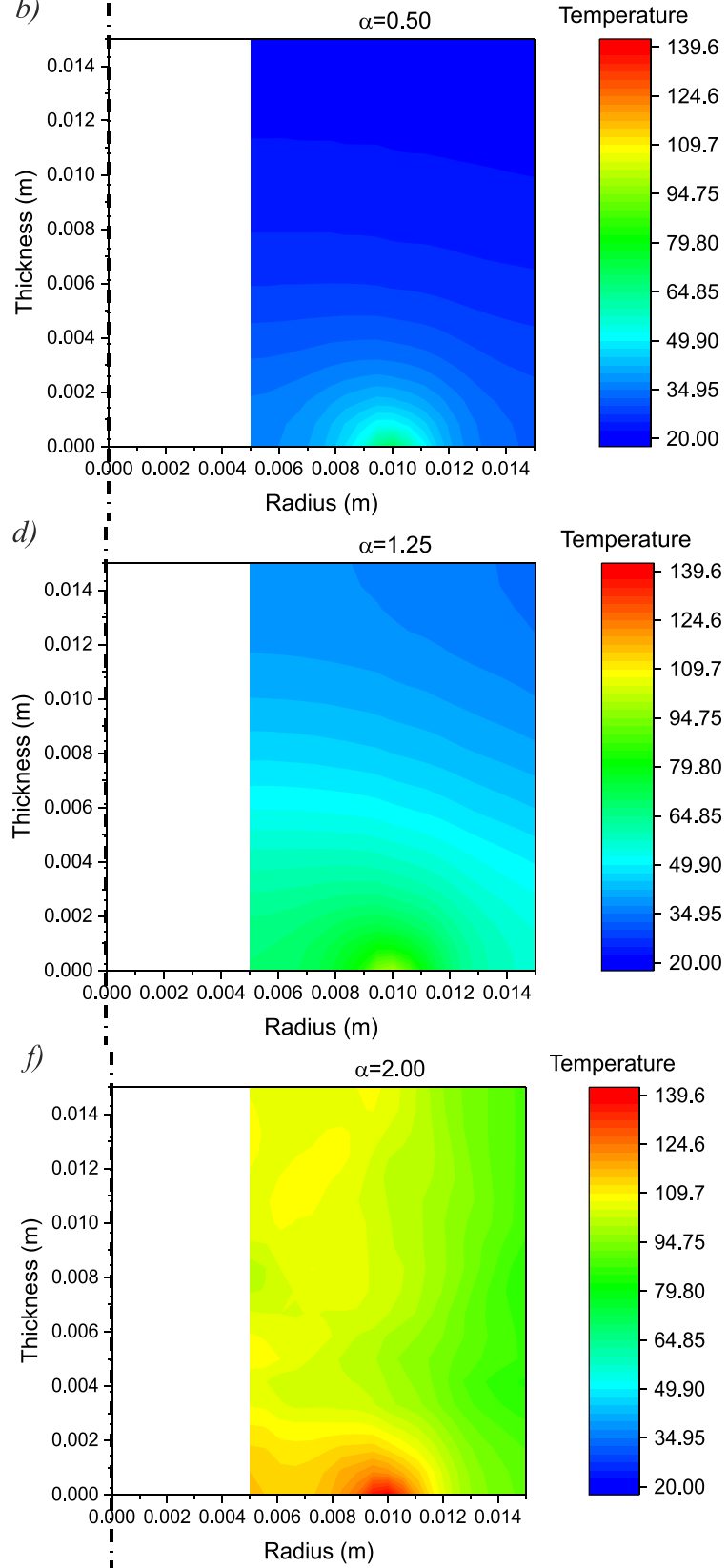

Fig. 5. The temperature distribution in the cross-section of the hollow cylinder for different $\alpha$.

Fig. 5 presents the temperature distributions for $t=10(s)$ and the order of fractional derivative $\alpha$ within $\alpha=0.1-2.0$. By analysing the collected results, it might be noticed that for $\alpha$ from the range of $0<\alpha<1.0$ the velocity of the overtemperature changes over time is low and does not exceed the values which are to be found for $\alpha=1$.

In the case of the order of derivative of the overtemperature over time with the value $\alpha$ from the range of $1.0<\alpha<2.0$, the temperature is approximately 1.5 times higher than for $\alpha=1$. In the case of $\alpha=2$ the so called ballistic heat conduction equation is to be found.

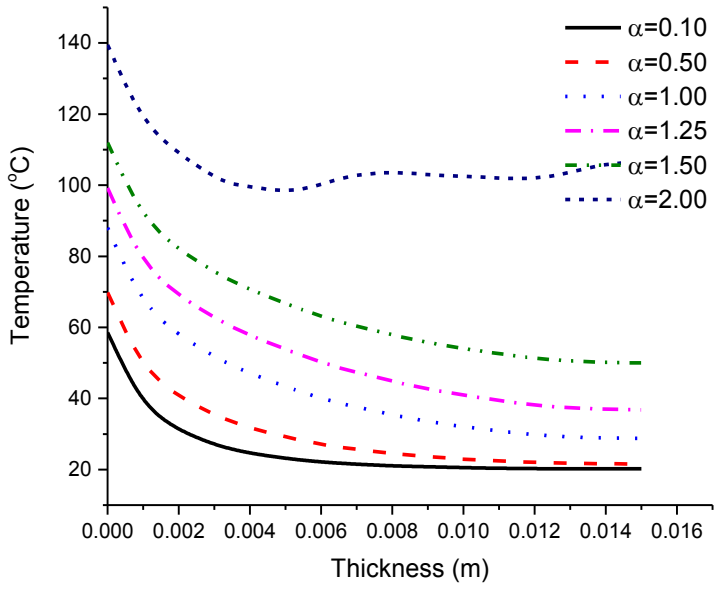

Fig. 6. The temperature distribution along the ring thickness on the radius $\bar{r}$ for different values of the equation $\operatorname{order} \alpha$. 


\section{Conclusion}

The paper presents the mathematical model of heat conduction for the hollow cylinder, where one front surface was treated with Gaussian-distributed heat flux. The results of the analytical solution of the mathematical model were presented in two aspects. The first one referred to the classical Fourier law (for $\alpha=1$ ), while the second one regarded applying the derivative of fractional order with respect to time (for $0<\alpha \leq 2$ ). The use of differential equations of fractional order expanded the possibilities of analysing the phenomenon of heat conduction in the hollow cylinder to a great extent. It is particularly visible in Fig. 6 which depicts the temperature distributions for $\mathrm{t}=10$ (s) in the form of a bundle of curves created for different values of the coefficient $0<\alpha \leq 2$, which is the order of fractional derivative. The change of the coefficient $\alpha$ from 1 to 2 illustrates the transition from the heat equation to the wave differential equation. The character of the latter is clearly visible in Fig. $5 \mathrm{f}$ in the form of "chaotic" temperature changes in the ring crosssection. It should be emphasized that the mathematical analysis using the differential equation of fractional order is useful to construct more detailed mathematical models describing the physical phenomena.

\section{References}

1. S. Blasiak, Int. J. Heat Mass Transfer 81 (2015), 90-102

2. S. D. Warbhe, J. J. Tripathi, K. C. Deshmukh et al., J. Heat Transfer 139(4) (2017), 44502

3. M. A. Ezzat, A. A. El-Bary, Int. J. Therm. Sci. 108 (2016), 62-69

4. P. A. Laski. In: Zolotarev I, Radolf V (eds) ENGINEERING MECHANICS 2016. ACAD SCI CZECH REPUBLIC, INST

THERMOMECHANICS, DOLEJSKOVA 5, PRAGUE 8, 182 00, CZECH REPUBLIC (2016), pp 350-353

5. S. Blasiak, J. E. Takosoglu, P. A. Laski, J THERM SCI TECH-JPN 9(2) (2014), JTST0011-JTST0011

6. J. Bochnia. In: Martsynkovskyy V, Zahorulko A (eds) XIIITH INTERNATIONAL SCIENTIFIC AND ENGINEERING CONFERENCE HERMETIC SEALING, VIBRATION RELIABILITY AND ECOLOGICAL SAFETY OF PUMP AND COMPRESSOR MACHINERYHERVICON-2011, vol 39. ELSEVIER SCIENCE BV, SARA BURGERHARTSTRAAT 25, PO BOX 211, 1000 AE AMSTERDAM, NETHERLANDS (2012), pp 98-110

7. J. E. Takosoglu. In: Zolotarev I, Radolf V (eds) ENGINEERING MECHANICS 2016. ACAD SCI CZECH REPUBLIC, INST THERMOMECHANICS, DOLEJSKOVA 5, PRAGUE 8, 182 00, CZECH REPUBLIC (2016), pp 546-549

8. S. Blasiak, Int. J. Heat Mass Transfer 100 (2016), 79-88
9. C. Marugán-Cruz, O. Flores, D. Santana et al., Int. J. Heat Mass Transfer 96 (2016), 256-266

10. Y. Z. Povstenko, Int. J. Diff. Eq. 2012(7) (2012), 113

11. Y. Z. Povstenko, Math. Prob. in Eng. 2014(3) (2014), 1-7

12. Y. Povstenko, Arch Appl Mech 82(3) (2012), 345362

13. Y. Povstenko, Entropy 15(10) (2013), 4122-4133

14. Y. Povstenko, Comm in App Ind. Math. 6(1) (2014)

15. H.-T. Qi, H.-Y. Xu, X.-W. Guo, Comput. Math. Appl. 66(5) (2013), 824-831

16. X. Jiang, M. Xu, Physica A 389(17) (2010), 3368 3374

17. W. E. Raslan, jtam (2016), 295

18. H. M. Youssef, Acta Mech 223(6) (2012), 12191231

19. X.-Y. Zhang, X.-F. Li, Eng. Fract. Mech. 171 (2017), 22-34

20. S. Rogosin, Mathematics 3(2) (2015), 368-381

21. H. J. Haubold, A. M. Mathai, R. K. Saxena, J. Appl. Math. 2011(3) (2011), 1-51 